\title{
Examination of quantitative methods for analyzing data from concept inventories
}

\author{
Eric Burkholder $\odot,{ }^{1}$ Cole Walsh, ${ }^{2}$ and N. G. Holmes ${ }^{2}$ \\ ${ }^{1}$ Department of Physics, Stanford University, Stanford, California 94305, USA \\ ${ }^{2}$ Laboratory of Atomic and Solid State Physics, Cornell University, Ithaca, New York 14853, USA
}

(Received 6 May 2020; accepted 18 June 2020; published 29 June 2020)

\begin{abstract}
Physics education research (PER) has long used concept inventories to investigate student learning over time and to compare performance across various student subpopulations. PER has traditionally used normalized gain to explore these questions but has begun to use established methods from other fields, including Cohen's $d$, multiple linear regression, and linear mixed effects models. The choice of analysis method for examining student learning gains in PER is a current subject of debate. We synthesize this debate here by focusing on the research questions and interpretations that can be drawn using various statistical tools. We focus particularly on how results can be interpreted through an equity lens and what questions can and cannot be answered using different methods. Our results demonstrate the importance of clearly defining a research question and using appropriate tools to answer that question.
\end{abstract}

DOI: 10.1103/PhysRevPhysEducRes.16.010141

\section{INTRODUCTION}

One of physics education research's greatest contributions to discipline-based education research (DBER) was the pioneering of the concept inventory $(\mathrm{CI})$ : a short, conceptual assessment administered at the beginning and end of a course to assess student learning. Researchers and instructors often use CIs to ask how much students learned, how effective was the instructor's pedagogy on various timescales, or how effective was the instructor's pedagogy for various student subpopulations. There are many different ways to quantify student learning and performance through these assessments, and active debates in the community about the appropriateness of various methods [1-6].

In this paper, we discuss the assumptions and limitations of various statistical methods and demonstrate how different methods actually answer distinct research questions. We examine tools commonly used in other fields of social science that the physics education research (PER) community has called for adoption, namely, Cohen's $d$ [2], multiple linear regression, and mixed effects models [4,5]. We also discuss normalized gain (and normalized change) [7], as it is a tool commonly used in PER and one currently under increased scrutiny [2].

The choice of analysis tool for evaluating CI data has important methodological implications for a current subject

Published by the American Physical Society under the terms of the Creative Commons Attribution 4.0 International license. Further distribution of this work must maintain attribution to the author(s) and the published article's title, journal citation, and DOI. of debate in PER: comparing learning between student populations and subpopulations [2,8-17]. Several studies, for example, have found that the size and direction of apparent performance differences between male and female students may depend on which metric one uses to measure that difference $[2,18,19]$. In this paper, we clarify how this conflict can be explained by considering the inherently different research questions that can be answered by each of these metrics.

These questions can be interpreted through an equity lens [3,20,21]. Quantitatively, we operationalize equity here based on the definitions in Refs. [3,20,21]:

1. Equity of individuality: students from a particular group improve in performance following an intervention. A group of students (e.g., students in a particular course, women, first-generation students of color) would exhibit equity of individuality if the group improved their CI scores, on average, following an intervention (that can be of any duration). This definition of equity, notably, does not compare students from different groups.

2. Equity of outcomes: students from different groups (e.g., courses with different instruction, gender, race or ethnicity) have the same level of performance, on average, at the end of an intervention. A class would exhibit equity of outcomes if students from different groups had the same CI score, on average, at the end of an intervention.

3. Equity of learning: students from different groups learn the same amount ignoring incoming preparation. A class would exhibit equity of learning if students from different groups (e.g., men and women) had the same raw change in scores, on average, following an intervention. This model of 


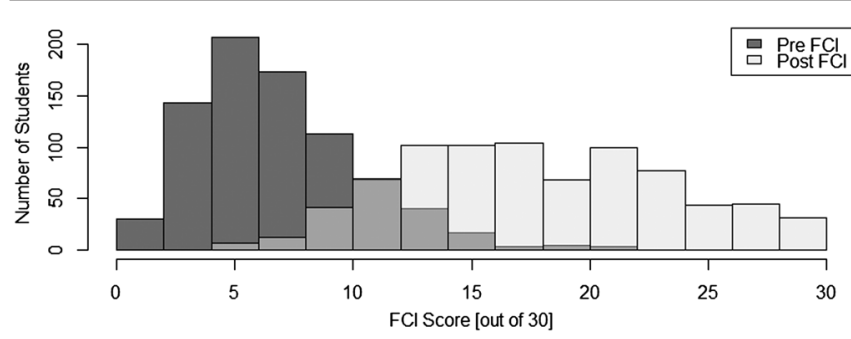

FIG. 1. Histogram of pretest scores (dark gray) and post-test scores (light gray) on the FCI for students at PCSSW. The histograms are overlapping, so the medium-gray scores in the middle are part of the pretest distribution.

equity preserves baseline differences in students' levels of performance.

We additionally define a fourth model of equity that is not explicitly covered by the three models above:

4. Equity of opportunity: students from different groups, if they had equivalent incoming performance, have the same expected performance following an intervention. A class would exhibit equity of opportunity if students from different groups had the same predicted post-test score (or, equivalently, change scores) for any given pretest score.

This fourth model of equity fills a gap in available research questions not covered by the three other models of equity by examining outcomes conditioned on baseline differences between students. Equity of opportunity can be viewed as a middle ground between equity of learning and equity of outcomes. Whereas equity of learning preserves differences in students' levels of performance and equity of outcomes eliminates differences, equity of opportunity reduces, but does not necessarily eliminate, differences. If equity of opportunity exists between two groups of students with different average pretest scores, then the change scores (i.e., difference in pre- and post-test scores, $x_{\text {post }}-x_{\text {pre }}$ ) will be larger for the lower performing group and we will approach equity of outcomes [22].

The type of equity one is interested in investigating will determine which quantitative methods are most appropriate for analyzing the CI data. As a case study to compare these different methods and demonstrate the research questions they can answer, we use an example dataset collected from 11th-grade high school physics courses at a public charter school in the southwestern U.S. (PCSSW). From 2006-2018, the Force Concept Inventory (FCI) [23] was administered as a pre- and post-test at the PCSSW to assess student learning. We have scores for a total of 803 students from 36 different courses across this 12-year period; there are no missing data. Matched pretest and post-test scores are plotted in Fig. 1 and appear similar to distributions seen in many university courses [24]. The average pretest score is 7.3 points (standard deviation 3.5 points) and the average post-test score is 18 points (standard deviation 5.6 points).

In the remainder of the paper, we use these data to evaluate the various analysis methods mentioned above.
The primary purpose of this paper is to add new perspectives on the benefits and limitations of each method and to serve as a guide for physics education researchers in future analyses of CI data. We assume the reader has some basic familiarity with $t$ tests and ANOVA. We recommend Ref. [25] for an introduction to these and other quantitative methods used in PER. In Sec. II, we discuss Cohen's $d$ as a method that can be used to answer equity of individuality, outcomes, and learning questions. In Sec. III, we discuss normalized gain and normalized change. These methods are typically used in PER to answer questions related to equity of opportunity. In Sec. IV, we discuss multiple linear regression (MLR) as a tool to answer questions related to all four equity models discussed above. In Sec. V, we discuss mixed effects models, which go beyond MLR to account for correlated measurements of the same quantity. We focus on the specific use of mixed effects models for repeated measures data where we can answer equity of individuality, outcomes, and learning questions, as with Cohen's $d$. We conclude the paper with summarizing comparisons between the various methods, including additional limitations.

\section{COHEN'S $d$}

Cohen's $d$ is a popular measure of effect size used in other social sciences [26] and medicine [27] that has recently gained attention in PER [2,26]. Cohen's $d$ is a measure of effect size that can be used to answer basic PER questions with CIs, such as did students learn anything at all (e.g., are scores at post-test higher than scores at pretest?) or do differences in performance exist between groups of students (e.g., do male and female students score differently at pretest?). Cohen's $d$ measures the magnitude of the difference in scores between two time points or two groups relative to the variability in the data (i.e., the difference in scores in units of standard deviations) [28]. Unlike $p$ values from $t$ tests, effect sizes, such as Cohen's $d$, can be compared across studies.

\section{A. Overview}

Cohen's $d$ is often reported in tandem with $t$ tests as a measure of the size of an effect (i.e., mean difference for paired samples or difference of means for independent samples). $t$ tests provide $p$ values that indicate the probability of observing a test statistic due to random chance alone, but $t$ tests are sample size dependent; with larger sample sizes, there is more power to detect effects. $t$-tests can be used, then, to determine the probability that an effect (of any size) exists, while Cohen's $d$ can be used to measure the size of this effect.

Cohen's $d$ for paired samples can be used to measure the size of the difference between a set of $N$ students' pre- and post-test scores. Cohen's $d$ is calculated as 


$$
d_{\text {paired }}=\frac{\frac{1}{N} \sum_{i} c^{i}}{s_{c}},
$$

where $c^{i}=x_{\text {post }}^{i}-x_{\text {pre }}^{i}$ is the change score for the ith student and $s_{c}$ is the standard deviation of students' change scores. Cohen's $d$ for independent samples, meanwhile, can be used to measure the size of the difference between sets of students at either pre- or post-test:

$$
d=\frac{\bar{x}_{1}-\bar{x}_{2}}{s_{p}},
$$

where $\bar{x}_{1}$ and $\bar{x}_{2}$ are the sample means for the two populations and $s_{p}$ is the pooled standard deviation:

$$
s_{p}=\sqrt{\frac{\left(n_{1}-1\right) s_{1}^{2}+\left(n_{2}-1\right) s_{2}^{2}}{n_{1}+n_{2}-2}},
$$

where $n_{1}$ and $n_{2}$ are the two independent sample sizes, and $s_{1}$ and $s_{2}$ are the independent sample standard deviations.

Interpreting Cohen's $d$ requires four assumptions be met (the same assumptions associated with running a $t$ test):

1. the observations in the sample are independent,

2. the dependent variable is numeric,

3. the population is normally distributed,

4. the variances are equal for the two groups (only for independent samples analysis).

Effect sizes for nonparametric tests (such as the HodgesLehmann estimator associated with the Wilcoxon signedrank test $[17,29]$ ) should be computed on heavily skewed data, while Cohen's $d$ can be modified to handle nonhomogeneous variances [30]. All of these assumptions are best checked visually.

\section{B. Sample analysis}

As identified above, Cohen's $d$ effect sizes can be used to compare CI scores for one group at two time points (equity of individuality), two groups at the same time point (equity of outcomes), or the change scores of two groups (equity of learning). For example, for the PCSSW dataset, we can answer questions related to equity of individuality by computing the Cohen's $d_{\text {paired }}$ effect size between all students' scores at pre- and post-test, which gives $d_{\text {paired }}=2.3 \pm 0.13$ (this range indicates a $95 \%$ confidence interval). This index indicates that students improved their scores by approximately 2.3 standard deviations by the end of the semester, on average. We could also answer equity of individuality questions by computing Cohen's $d$ for a subgroup's pre- and post-test score. Cohen's $d$ for male students from pre- to post-test is $d_{\text {paired,Male }}=2.4 \pm 0.23$, while for female students it is $d_{\text {paired,Female }}=2.5 \pm 0.16$, indicating that both groups improved their performance. We remind the reader that $d_{\text {paired,Male }}$ is based only on male students' scores and $d_{\text {paired,Female }}$ is based only on female students' scores. The values are still comparable because it is a difference in means scaled by the standard deviation, the idea behind Cohen's $d$ is that you can compute for different populations and still have a comparable effect size. We can answer equity of outcomes questions by computing the Cohen's $d$ effect sizes between, for example, male and female students at pretest and at post-test. In doing so, we find at pretest $d_{\text {pre }}=0.89 \pm 0.16$, indicating that male students' pretest scores are, on average, 0.89 standard deviations higher than those of female students. At post-test, the difference is slightly smaller, $d_{\text {post }}=0.78 \pm 0.16$. To investigate equity of learning, we can also compute Cohen's $d$ for the difference in change scores between male and female students. We find $d_{\text {change }}=0.25 \pm 0.15$, indicating that the change in scores is indeed larger for male students by about 0.25 standard deviations.

\section{Limitations}

Cohen's $d$ is only useful for comparing across two time points or two groups. With more than two time points or groups, we could calculate a Cohen's $d$ for each pairwise comparison, but this can lead to "multiple comparisons" issues: When many different comparisons are made, it becomes statistically more likely to measure large effects simply due to chance alone. Instead, we can turn to effect sizes from analysis of variance (ANOVA), which can compare the performance of two or more groups. $\eta^{2}$ and $\eta_{\text {partial }}^{2}$ are two commonly estimated effect sizes for ANOVA.

A significant limitation of Cohen's $d$ is that the analysis cannot control for other variables. ANOVA can control for other categorical variables, but neither Cohen's $d$ or ANOVA can control for other continuous variables, such as students' pretest scores. This limitation places significant constraints on the possible questions that can be answered using Cohen's $d$. Cohen's $d$ and ANOVA can be used to determine whether a difference exists in students' pretest scores, but cannot use this information when examining students' post-test or change scores. Cohen's $d$ cannot, then, be used to answer questions related to equity of opportunity and whether the difference in post-test or change scores between men and women in the PCSSW can be explained by a difference in pretest scores.

\section{Why control for pretest scores?}

One of the key features of CIs is that they allow us to compare the impacts of instruction at multiple institutions with different populations of students, using a standardized measurement. The variability between our participants means we often want to account for systematic differences in students' preparation. The argument is that, because CIs are bounded (students cannot score lower than $0 \%$ or higher than $100 \%$ ), an increase in test scores of $10 \%$ (or 0.2 standard deviations) is conceptually different if the student started with a pretest score of $10 \%$ or $90 \%$. For the former, 
$10 \%$ (or 0.2 standard deviations) is just a small amount of what they could have learned. For the latter, they could only improve their score by $10 \%$.

Ultimately, we seek to control for incoming performance because of regression to the mean [22,31-33]. In addition to being bounded, the CIs we use are imperfect; there is always some associated measurement error when measuring student performance. Students who score high at pretest could be highly skilled students or they could be average or below average students who scored higher due to chance [33]. If this subset of students (who score high at pretest) were to take the assessment again at post-test, then the highly skilled students could score lower due to chance, while the below average students who scored high on the pretest would have to be lucky a second time in order to remain at the top of the class. While this scenario assumes that the hierarchy of students' skills is unchanged between tests (which is unrealistic), it illustrates that student improvement on assessments is related to both changes in skill and luck. This well-known concept of regression to the mean forces us to consider students' baseline performance when evaluating learning gains $[22,32]$.

Regression to the mean has practical implications for how we interpret quantitative analyses of CI data. Consider the following:

In the previous analysis, male students scored noticeably higher at pretest than female students $\left(d_{\text {pre }}=0.89\right)$. At post-test, however, the difference in performance had reduced to $d_{\text {post }}=0.78$.

We could interpret this scenario to mean that our instruction was very helpful to female students and/or not as helpful to male students, but neither interpretation is strictly true. Some of the decrease in performance difference between male and female students can likely be explained by the effect of instruction, but some is likely due to regression to the mean. We must refrain, then, from making claims about instruction without accounting for baseline differences across students [22].

Hake identified the issue of regression to the mean when developing a quantity called normalized gain [7], which we will discuss next. Ultimately, for research questions focused on equity of outcomes and equity of learning, regression to the mean impacts our interpretations of the results, as post-test and change scores can be different simply because the pretest scores of students in different groups are different.

\section{NORMALIZED GAIN AND NORMALIZED CHANGE}

As discussed above, one of the limitations of Cohen's $d$ and effect sizes associated with ANOVA are that they do not control for baseline differences in student populations. Normalized gain and normalized change were developed to address this shortcoming.

\section{A. Overview}

Hake introduced normalized gain as a way to measure learning that could be compared across classes with different levels of incoming physics knowledge [7] (i.e., equity of opportunity). Normalized gain is the change in a class's average score between pre- and post-test divided by the maximum possible change:

$$
g=\frac{\bar{x}_{\text {post }}-\bar{x}_{\text {pre }}}{\max (x)-\bar{x}_{\text {pre }}}
$$

where $\bar{x}_{\text {post }}$ is the average class post-test score, $\bar{x}_{\text {pre }}$ is the average class pretest score, and $\max (x)$ is the maximum score on the assessment. Loosely speaking, normalized gain is often interpreted as the average fraction of concepts learned by a class that was not known at the beginning of the course.

Though normalized gain appears to answer questions related to equity of opportunity, it cannot address this model of equity exactly. We discuss equity of opportunity in more detail in Sec. IV C 1, but, in general, groups will have different normalized gains under the conditions of equity of opportunity defined here; two groups with identical post-test or change scores for any given pretest score will generally have different normalized gains. Within our equity framework, normalized gain can only be used to evaluate equity of individuality.

\section{B. Sample analysis}

We calculated normalized gain at the PCSSW, following the procedure in Ref. [16], by first finding the normalized gain for each of the 36 courses in our dataset and then taking the average of these 36 values. We find that, on average, students at the PCSSW learned about $48 \%$ of the material that they did not know at the outset of the course. We can also compute the average normalized gain separately for male and female students at the PCSSW: $\bar{g}_{\text {Female }}=0.45$ and $\bar{g}_{\text {Male }}=0.58$ (see: Fig. 2). We find that female students learn just under half the concepts they did not know at the beginning, while male students learn just over half the concepts they did not know at the beginning.

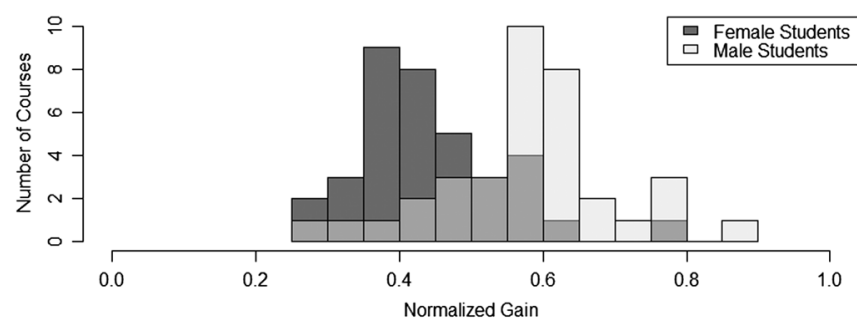

FIG. 2. Histogram of normalized gain for male students (light) and female students (dark) in each class at PCSSW. 


\section{Limitations}

We cannot strictly compare these values of normalized gain for male and female students through the lens of equity of opportunity, but the discrepancy between these results and those presented in Sec. II B highlight a key difference between normalized gain and Cohen's $d$. Our results from Sec. II B, when we calculated Cohen's $d$ from pre-to posttest for male students, $d_{\text {paired,Male }}$, and for female students, $d_{\text {paired,Female }}$, suggested that male students saw approximately the same improvement from pretest to post-test, on average, as female students. Our results here, however, indicate that normalized gain is much higher for male students. We can reconcile this disagreement in results by considering the different research questions being answered. Cohen's $d$ examines post-test or change scores without regard for students' pretest scores, whereas normalized gain takes into account students' pretest scores when examining change scores. Cohen's $d$ answers equity of outcomes and learning questions, but normalized gain answers a different research question, related to equity of opportunity. Some researchers have recently argued that Cohen's $d$ should be used instead of normalized gain [2]. We agree with other researchers (e.g., Ref. [1]), however, that Cohen's $d$ is not an appropriate alternative to normalized gain because it does not attempt to answer the same question.

Compared with many statistical tools, normalized gain has an intuitive interpretation and is widely used by the PER community. The statistical properties of normalized gain, however, limit the statistical interpretations that we can make. There is a gross asymmetry in the range of normalized gain $[g \in(-\infty, 1]]$ that has led some to argue that this statistic is "biased" [2,18,34]. Marx and Cummings introduced an alternative to normalized gain to mitigate $g$ 's asymmetry, called normalized change [34]:

$$
c_{\text {norm }}= \begin{cases}\frac{\bar{x}_{\text {post }}-\bar{x}_{\text {pre }}}{\max (x)-\bar{x}_{\text {pre }}}, & \bar{x}_{\text {post }}>\bar{x}_{\text {pre }} \\ 0, & \bar{x}_{\text {post }}=\bar{x}_{\text {pre }} \\ \frac{\bar{x}_{\text {post }}-\bar{x}_{\text {pre }}}{\bar{x}_{\text {pre }}}, & \bar{x}_{\text {post }}<\bar{x}_{\text {pre }}\end{cases}
$$

This statistic has a symmetric range $c_{\text {norm }} \in[-1,1]$, making it more attractive and potentially mitigating low prescore bias. Typically, analyses using normalized change show only minor quantitative differences from analyses using normalized gain [35].

A more salient issue with normalized gain is the difficulty with which it can be compared across two different courses. Suppose we had two classes with the same pretest, using the same instructional strategy, but with very different post-test averages. If one class had 10 students and another had 1000 students, how do we interpret the effectiveness of the instructional strategy? The normalized gains will be very different for each class, but we do not have information about the reliability of the measures (e.g., standard error). Cohen's $d$ and effect sizes through ANOVA, in contrast, have well-defined statistical properties that make these tools more suitable for research analyses.

Both $g$ and $c_{\text {norm }}$ suffer from statistical issues beyond the symmetry of the distributions and the lack of a standard error for individual measurements of $g$. We briefly summarize these issues in Ref. [36]. The critical issue is that the population distribution of normalized gain (the theoretical distribution of $g$ over all courses) for the population of classes is so heavy tailed (see the skew in Fig. 2, particularly for groups of female students) that its mean and variance are undefined. That is, integrating the population distribution of normalized gain over its range does not converge. Thus, the central limit theorem does not guarantee that the sample mean will be normally distributed for even a large number of samples. Hypothesis testing is thus not possible using typical parametric methods, which assume an underlying normal distribution of the test statistics. This places significant limitations on our ability to interpret normalized gain measures: how do we know if a value of normalized gain is statistically different from another if there is no way to determine its mean or variance? We note that one could, for example, still calculate a sample mean of normalized gain (e.g., Fig. 2), but the population mean over all hypothetical samples of normalized gain is infinite.

Ultimately, normalized gain and normalized change are statistically quite problematic. These tools were developed to answer questions related to equity of opportunity, namely, the effect of instruction on student CI performance independent of students' incoming preparation, but fail in this endeavor. Cohen's $d$ and ANOVA cannot answer equity of opportunity questions either and are not, then, suitable alternatives to normalized gain and normalized change. We therefore turn to MLR, which can answer equity of opportunity questions; namely, do students learn the same amount, accounting for where they started? MLR can also answer other equity questions, while simultaneously controlling for variables other than pretest score, which is impossible with normalized gain or change, Cohen's $d$, and ANOVA.

\section{MLR}

Multiple linear regression is a versatile tool that can be used to answer any of our types of equity research questions, effectively replacing Cohen's $d$, ANOVA, and normalized gain or change. MLR can also extend on these methods and control for many variables simultaneously, including pretest score.

\section{A. Overview}

MLR measures the proportion of variance in a dependent variable explained by separate independent variables and 
can examine the relative contributions of the independent variables to a predicted outcome. MLR aims to fit a linear relationship between variables, uses the data to estimate the intercept and slope(s) of the line, and tests hypotheses that these coefficients are nonzero. Typically, the regression coefficients (i.e., slopes and intercepts) are estimated using the method of ordinary least squares, which minimizes the squared difference between the estimated and true values of the independent variable $|\epsilon|^{2}$. An overview of the detailed mathematics may be found in Ref. [36]. For CI data, MLR can be used to measure the size of the effects of independent variables on post-test or change scores.

For example, MLR can simultaneously measure the effect of gender and physics preparation (e.g., CI pretest score) on course outcomes (e.g., CI post-test score). With MLR, we can measure the effect of each independent variable in units of the dependent variable, allowing us to determine the average predicted difference between groups of students while controlling for the other variables, including pretest scores. In this example, our MLR model can be written as

$$
x_{\text {post }}=\beta_{0}+\beta_{1} x_{\text {pre }}+\beta_{2} \text { Gender }+\epsilon
$$

The regression coefficients $\beta_{1}$ and $\beta_{2}$ may be interpreted as the effect sizes for pretest score and gender, respectively. The coefficient $\beta_{1}$ indicates the average change in post-test score for each unit change in pretest score. The coefficient $\beta_{2}$ indicates the average difference in predicted post-test scores between male and female students who have the same pretest score [37]. $\beta_{2}$ can be interpreted through the lens of equity of opportunity. If $\beta_{2}=0$ in Eq. (6), both male and female students have the same predicted post-test score for any given pretest score (assuming no interactions or higher-order terms).

MLR does not make any assumptions about the distribution of either the predictors (independent variables) or the response (dependent variables) and is thus applicable to data with a wider range of distributions than simple $t$ tests and ANOVA. In order for MLR parameter estimates to be unbiased and most efficient (require the smallest number of data points to estimate to a given accuracy), there are five assumptions that must be met [37]:

1. Each observation of post-test score is independent from the other observations but drawn from the same probability distribution with respect to all independent variables (e.g., gender and pretest score). Practically speaking this means that the scores of one student should be independent of the scores of another student.

2. All independent and dependent variables have finite, nonzero fourth moments (e.g., $\left.\mathbb{E}\left[\left(x_{\text {post }}-\bar{x}_{\text {post }}\right)^{4}\right]\right)$. This is a mathematical constraint requiring that the tails of the distribution of scores are not too heavy.
3. The average residual error for any given value of the independent variables is zero (e.g., $\mathbb{E}\left[\epsilon \mid x_{\text {pre }}\right.$, Gender $\left.]=0\right)$. That is, $\epsilon$ should not be correlated with the independent variables.

4. The errors are constant across different values of the independent variables (e.g., $\operatorname{Var}\left[\epsilon \mid x_{\text {pre }}\right.$, Gender $]=\sigma^{2} \mathbb{I}$, where $\mathbb{I}$ is the identity tensor and $\sigma^{2}$ is the variance of the residuals). That is, $\epsilon$ should not be different for different values of the independent variables.

5. The independent variables are not linearly dependent. Violation of any one of these assumptions biases the estimates of the regression coefficients or inflates the standard errors of the coefficients [33]. Thus, it is important when using MLR to check that these assumptions are not violated before drawing conclusions based on the data. These assumptions can be checked through visual inspection of the residuals, as shown in Ref. [36]. More detail about the consequences of violating each assumption may be found in Ref. [36].

\section{B. Pedagogical notes}

We have three subtle notes about MLR that may be useful to an interested reader and help with understanding statistical tool choices in PER.

\section{MLR versus ANCOVA}

The model used by MLR is identical to that used in analysis of covariance (ANCOVA), but the estimated effect sizes are different. The effect sizes used with ANOVA (i.e., $\eta^{2}$ and $\eta_{\text {partial }}^{2}$ ) are typically applied to ANCOVA. $\eta^{2}$ is calculated the same way as $R^{2}$ for MLR and can be interpreted in much the same way. The only difference is $\eta^{2}$ in $\mathrm{AN}(\mathrm{C}) \mathrm{OVA}$ represents the proportion of variance explained by each variable, while $R^{2}$ in MLR represents the proportion of variance explained by all of the variables together. As mentioned in the previous section, coefficients from MLR can be interpreted as the average difference in post-test score for each level of a categorical variable, or the average change in post-test score for each unit of a continuous variable. These coefficients, therefore, are more intuitive interpretations of the size of the effects in these analyses (in terms of actual student performance).

\section{Modeling post-test scores versus change scores}

For many PER questions, we seek to understand how performance has changed, rather than where students "end up." That is, we could have modeled students' change scores as the outcome variable instead of modeling for post-test scores in Eq. (6). Some simple algebra can demonstrate that these models are actually isomorphic $[38,39]$. Subtracting $x_{\text {pre }}$ from both sides of Eq. (6), we arrive at 


$$
x_{\text {post }}-x_{\text {pre }}=\beta_{0}+\left(\beta_{1}-1\right) x_{\text {pre }}+\beta_{2} \text { Gender }+\epsilon
$$

The left-hand side of Eq. (7) represents students' change scores. The parameter estimates for this fitted model are the same as for the model represented by Eq. (6), save for the coefficient for pretest scores being offset by one. Thus, the effect size of gender on change scores controlling for pretest scores is identical to the effect size of gender on post-test scores controlling for pretest scores. This isomorphism between models allows us to frame equity of opportunity in two equivalent ways: students from different groups, if they had equivalent incoming performance, have the same predicted (i) post-test score and (ii) change score.

\section{Modeling raw versus standardized scores}

Equation (6) gives effect sizes in units of raw points. For example, $\beta_{2}$ in Eq. (6) represents the average difference in raw post-test scores between male and female students after controlling for pretest scores. We often seek a standardized measure of effect size that is independent of the scale of an assessment (see Sec. II), such that we can interpret performance on different CIs. Common practice is to scale pre- and post-test scores independently to have mean zero and standard deviation one (i.e., convert to $z$ scores). Equation (6) can then be recast as

$$
z_{\text {post }}=\beta_{0}+\beta_{1} z_{\text {pre }}+\beta_{2} \text { Gender }+\epsilon,
$$

where $z_{\text {pre }}$ and $z_{\text {post }}$ are a student's scaled pre- and post-test scores, respectively. $\beta_{2}$ now represents the average difference in post-test scores between men and women in units of post-test standard deviations (rather than in units of score), again controlling for pretest scores. The advantage of effect sizes calculated in this way is that they can be compared across assessments with different total points and are on roughly the same scale as Cohen's $d$. Continuous and categorical independent variables within the same model can also be more easily compared.

\section{Sample analysis and limitations}

We apply MLR to the PCSSW dataset in two parts, focusing first on using MLR to answer equity of opportunity questions (where we control for pretest scores). Then we demonstrate how to use MLR to answer equity of individuality, outcomes, or learning questions similar to Cohen's $d$.

\section{Using MLR to control for incoming preparation}

We fit the model represented by Eqs. (6) and (8) to our PCSSW dataset. The results are summarized in Model 1 in Table I. We examine $\beta_{2}$ to probe equity of opportunity, comparing the post-test scores of male and female students with the same pretest scores. The average post-test $z$ score for a male student with an average pretest score $\left(z_{\text {pre }}=0\right)$ is 0.28 (15 points, i.e., $\beta_{0}$ ). The average post-test $z$ score for female students with an average pretest score is -0.12 (13 points, found by adding $\beta_{0}+\beta_{2}$ ). Thus, female students receive lower post-test scores, on average, than male students (by 2 points), even if they have the same pretest score.

Something often omitted from analyses using MLR, and impossible to examine using normalized gain, is the possibility of an interaction between gender and pretest score that allows both the slope and intercept to vary by gender. With this additional term, our model becomes

$x_{\text {post }}=\beta_{0}+\beta_{1} x_{\text {pre }}+\beta_{2}$ Gender $+\beta_{3}$ Gender $\times x_{\text {pre }}+\epsilon$.

This interaction term allows us to probe equity of opportunity questions further: namely, does the relationship between

TABLE I. Raw and standardized coefficients for two MLR models using the PCSSW dataset. Model 2 includes an interaction term between pretest score and gender, allowing the relationship between pretest and post-test scores to vary across gender. There is

\begin{tabular}{|c|c|c|c|c|c|c|}
\hline & \multicolumn{6}{|c|}{ Dependent variable Post-test score } \\
\hline & \multicolumn{3}{|c|}{ Model 1} & \multicolumn{3}{|c|}{ Model 2} \\
\hline & $\begin{array}{l}\text { Raw } \\
\text { coefficient }\end{array}$ & $\begin{array}{l}\text { Standardized } \\
\text { coefficient }\end{array}$ & $p$ value & $\begin{array}{l}\text { Raw } \\
\text { coefficient }\end{array}$ & $\begin{array}{l}\text { Standardized } \\
\text { coefficient }\end{array}$ & $p$ value \\
\hline Constant $\left(\beta_{0}\right)$ & $\begin{array}{l}15.0 \\
(0.58)\end{array}$ & $\begin{array}{c}0.28 \\
(0.058)\end{array}$ & $<0.001$ & $\begin{array}{l}14.0 \\
(0.79)\end{array}$ & $\begin{array}{c}0.24 \\
(0.048)\end{array}$ & $<0.001$ \\
\hline Pretest score $\left(\beta_{1}\right)$ & $\begin{array}{c}0.66 \\
(0.053)\end{array}$ & $\begin{array}{c}0.41 \\
(0.033)\end{array}$ & $<0.001$ & $\begin{array}{c}0.76 \\
(0.078)\end{array}$ & $\begin{array}{c}0.48 \\
(0.033)\end{array}$ & $<0.001$ \\
\hline Gender (Female; $\left.\beta_{2}\right)$ & $\begin{array}{l}-2.23 \\
(0.40)\end{array}$ & $\begin{array}{l}-0.40 \\
(0.072)\end{array}$ & $<0.001$ & $\begin{array}{l}-0.66 \\
(0.094)\end{array}$ & $\begin{array}{l}-0.37 \\
(0.073)\end{array}$ & $<0.001$ \\
\hline Pretest score $\times$ Gender $($ Female $)\left(\beta_{3}\right)$ & & & & $\begin{array}{c}-0.20 \\
(0.10)\end{array}$ & $\begin{array}{l}-0.12 \\
(0.066)\end{array}$ & 0.063 \\
\hline AIC & & 4820 & & & 4819 & \\
\hline$R_{\text {adj }}^{2}$ & & 0.26 & & & 0.26 & \\
\hline
\end{tabular}
essentially no difference in AIC or $R^{2}$ between the two models, so the effect of the interaction term is nonsignificant. 
post-test and pretest vary for different subgroups of students? We summarize the results in Model 2 in Table I. In this model, the average post-test score for male students with average pretest scores $\left(z_{\text {pre }}=0\right)$ is $\beta_{0}=0.24$ (14 points) and the average post-test score for female students with average pretest scores $\left(z_{\text {pre }}=0\right)$ is $\beta_{0}+\beta_{2}=-0.13$ (13 points). Furthermore, male students see a 0.48 standard deviation increase in post-test score per standard deviation increase in pretest score $\left(\beta_{1}\right)$, while female students only see a 0.36 standard deviation increase in post-test score for the same increase in pretest score $\left(\beta_{1}+\beta_{3}\right)$. This means that the difference between male and female students' post-test scores will be different for different pretest scores. Female students average pretest score is $z_{\text {pre }}=-0.25$, at which point the model predicts a difference of 1.9 points between male and female students' post-test scores. The difference in scores at post-test is smaller for lower pretest scores and larger for higher pretest scores.

To decide whether this interaction term is significant or meaningful is to see if the quality of the model fit changes when terms are added to the regression equation. To do this, we compare the Akaike information criterion (AIC) for each model. The AIC balances a models likelihood function, which describes how plausible a model with the given parameters is, with the number of parameters being fit to encourage the choice of a parsimonious model-one that is a likely explanation for the data, but not overfit. When comparing two models, the model with the lower AIC is the better model. The AIC for the model that includes gender and pretest score as predictors of posttest score is 4820 . To three significant figures, the value of AIC is the same for the model including the interaction term (4819). Because there is essentially no difference in AIC, we would conclude that there is no effect of the interaction term. For more information on interpreting AIC values, see Ref. [40]. Because the models are nested-the model including the interaction term contains all of the independent variables contained in the model with no interaction term we can use ANOVA to test if the difference is statistically significant. ANOVA tells us that this difference is not statistically significant $F(1,799)=3.46$, $p=0.063$; the interaction term only marginally improves the fit of the model. The adjusted $R^{2}$ value for the models with and without the interaction term is about $26 \%$ in both cases. Given that the regression coefficient, change in $R^{2}$, and change in AIC are small, and that the term is only marginally significant, we argue that there is no practical significance to the interaction term. Qualitatively, this suggests that there is a gender gap in post-test scores for students with the same pretest score, but that the size of this gap does not depend significantly on pretest score.

\section{Using MLR without controlling for incoming preparation}

MLR can also reproduce results calculated using Cohen's $d$ with the PCSSW dataset to answer equity of individuality, outcomes, and learning questions. Consider the smaller MLR models:

$$
\begin{aligned}
& x_{\text {pre }}=\beta_{0}^{\prime}+\beta_{1}^{\prime} \text { Gender }, \\
& x_{\text {post }}=\beta_{2}^{\prime}+\beta_{3}^{\prime} \text { Gender } .
\end{aligned}
$$

We present the results of these fitted models in Table II. We find that male students are about 0.57 standard deviations above average at pretest (average score 9.3 points), while female students are about 0.26 standard deviations below average (i.e., $\beta_{0}^{\prime}+\beta_{1}^{\prime}$; average score 6.4 points). At posttest, we find male students are still above average, but the difference in scores between male and female students is slightly smaller. The standardized estimates of $\beta_{1}^{\prime}$ and $\beta_{3}^{\prime}$ are similar to the corresponding values of Cohen's $d$ from Sec. II $\left(d_{\text {pre }}=0.89\right.$ and $\left.d_{\text {post }}=0.78\right)$; the small quantitative differences between the regression parameter estimates and Cohen's $d$ are related to how standard deviations are pooled in the calculation of Cohen's $d$.

Thus, MLR is a viable alternative for answering questions typically solved with Cohen's $d$ or normalized gain or change. If there is a natural structure present in the data (e.g., students are nested within different classes), however, then observations of students may not be truly independent, violating assumption 1 above. MLR is also limited in

TABLE II. Raw and standardized coefficients for two simple linear regression models with PCSSW dataset and gender as the only explanatory variable. The first model uses pretest score as the dependent variable, while the second model uses post-test score as the dependent variable. $t$ statistics and $p$ values are identical to those obtained from independent samples $t$ tests or repeated measures analysis. Standardized coefficient estimates are similar to those obtained using Cohen's $d$, while raw coefficients are identical to those

\begin{tabular}{|c|c|c|c|c|c|c|}
\hline & \multicolumn{3}{|c|}{ Dependent variable Pretest score } & \multicolumn{3}{|c|}{ Dependent variable Post-test score } \\
\hline & Raw coefficient & Standardized coefficient & $p$ value & Raw coefficient & Standardized coefficient & $p$ value \\
\hline Constant $\left(\beta_{0}^{\prime}, \beta_{2}^{\prime}\right)$ & $\begin{array}{c}9.3 \\
(0.21)\end{array}$ & $\begin{array}{c}0.57 \\
(0.059)\end{array}$ & $<0.001$ & $\begin{array}{c}21.1 \\
(0.34)\end{array}$ & $\begin{array}{c}0.51 \\
(0.060)\end{array}$ & $<0.001$ \\
\hline Gender (Female) $\left(\beta_{1}^{\prime}, \beta_{3}^{\prime}\right)$ & $\begin{array}{l}-2.9 \\
(0.25)\end{array}$ & $\begin{array}{l}-0.83 \\
(0.071)\end{array}$ & $<0.001$ & $\begin{array}{l}-4.1 \\
(0.41)\end{array}$ & $\begin{array}{l}-0.73 \\
(0.072)\end{array}$ & $<0.001$ \\
\hline
\end{tabular}
obtained from repeated measures analysis. 
evaluating data across three or more time points. Both of these problems can be addressed by mixed effects models, discussed in Sec. V.

\section{MIXED EFFECTS MODELS OF REPEATED MEASURES DATA}

In this section, we introduce mixed effects models, which can go beyond MLR to account for correlations among observations and multiple time points. We give a brief overview of this technique in Sec. VA and focus on the particular use of this tool with repeated measures data (data which are taken at multiple time points). In Sec. V B we use our PCSSW dataset to illustrate how mixed effects modeling of repeated measures can replicate MLR that do not control for pretest scores to answer equity of individuality, outcomes, and learning questions. For a more complete discussion of how to use mixed effects models of repeated measures, we refer the reader to Ref. [5].

\section{A. Overview}

Mixed effects models are gaining traction in PER as a tool for dealing with data from multiple sources such that the error term in Eq. (8) may be different for students in different courses, schools, or years of instruction $[4,8,9,12,19]$. Mixed effects models can also be used to analyze repeated measures data. Mixed effects models in these situations take on a particular form. Analysis of repeated measures with mixed effects models the outcome variable (e.g., CI score) as a function of fixed effects, the average effects for all students over time, and random effects that describe unobserved, time-invariant differences between students [37]. By controlling for random effects, we ensure that our measure of student performance does not depend on time-invariant student effects (such as socialpsychological factors) [6]. Mathematically, the model of interest is

$$
x=\beta_{0}+\beta_{1} \text { Test }+\gamma_{1} Z+\epsilon,
$$

where the binary variable Test (which is zero for the pretest and one for the post-test in the case of the PCSSW data, but could be a multilevel variable if one was investigating, say, differences in homework score over the duration of a course) is the fixed effect, the random effect is $Z$, and the regression residual $\epsilon$ measures the difference between the actual scores and the scores predicted by the linear relation. Equation (11) can be written equivalently as

$$
x=\alpha+\beta_{1} \text { Test }+\epsilon,
$$

where $\alpha=\beta_{0}+\gamma_{1} Z$ is effectively the intercept of the model for each student. This model portions the variation in scores (pretest and post-test) into a piece that can be explained by the fixed effects (in this case the variance in when the test was administered) and the random effects (in this case, the variance in the students who are taking the test).

Mixed effects models make some of the same assumptions as MLR, while also assuming that there is a hierarchical structure to the data (e.g., tests nested within students or students nested within classes). Mixed effects models are well suited to answer equity of individuality, outcomes, and learning questions. For example, they allow one to simultaneously compute the difference between male and female students' pretest scores, post-test scores, and change scores. Such analysis would otherwise require several MLR analyses [as in Eqs. (10a) and (10b)], or multiple calculations of Cohen's $d$. Thus, mixed effects models of repeated measures data answer the same research questions as Cohen's $d$ and ANOVA: namely, is there an overall difference in student performance ignoring performance at other time points? Mixed effects models cannot be used to answer equity of opportunity questions. That is, these models cannot compute performance differences at a later time point for students with equivalent scores at an earlier time point.

\section{B. Sample analysis and limitations}

In this analysis, we seek to answer questions related to equity of individuality, learning, and outcomes: do scores improve over time and is performance different for male and female students at various time points? We are interested in fixed effects of time (pretest vs post-test), gender, and the interaction between time and gender. We model the random effects as different students. This model can be written as

$$
\begin{aligned}
\text { Score }= & \beta_{0}+\beta_{1} \text { Test }+\beta_{2} \text { Gender } \\
& +\beta_{3} \text { Test } \times \text { Gender }+\gamma_{1} Z+\epsilon .
\end{aligned}
$$

We present the results of this fitted model in Table III, using both raw and standardized coefficients. Unlike in Sec. IV, we scale pretest and post-test scores; that is, instead of converting pretest scores to $z$ scores and then post-test scores to $z$ scores, we group both together and convert all scores to $z$ scores using the mean and standard deviation of all scores. We report two goodness-of-fit measures: conditional and marginal $R^{2}$. The marginal $R^{2}$ value is the proportion of variance explained by the fixed effects, and the conditional $R^{2}$ value is the proportion of variance explained by both fixed and random effects (thus, a measure of the size of the random effect would be the difference in these two $R^{2}$ values). $p$ values are computed by loading the lmerTest package [41]. We note that there is some debate over how $p$ values should be estimated from mixed effects models. Other ways to determine the significance of fixed effects would be likelihood ratio tests, or 
TABLE III. Raw and standardized coefficients from mixed effects analysis with PCSSW dataset. Raw coefficients, $t$ statistics, and $p$ values are identical to those obtained from simple linear regression with gender as the only explanatory variable.

\begin{tabular}{lccc}
\hline \hline & \multicolumn{3}{c}{ Dependent variable Score } \\
\cline { 2 - 4 } & $\begin{array}{c}\text { Raw } \\
\text { coefficient }\end{array}$ & $\begin{array}{c}\text { Standardized } \\
\text { coefficient }\end{array}$ & $p$ value \\
\hline Constant & 9.30 & -0.48 & $<0.001$ \\
& $(0.24)$ & $(0.046)$ & \\
Test & 11.8 & 1.6 & $<0.001$ \\
(Post-test) & $(0.28)$ & $(0.044)$ & \\
Gender (Female) & -2.9 & -0.40 & $<0.001$ \\
& $(0.29)$ & $(0.046)$ & \\
Test (Post-test) & -1.2 & -0.17 & $<0.01$ \\
$\quad \times$ Gender (Female) & $(0.33)$ & $(0.053)$ & \\
Marginal $R^{2}$ & 0.63 & 0.63 & \\
Conditional $R^{2}$ & 0.76 & 0.76 & \\
\hline \hline
\end{tabular}

to use the AIC to compare models with and without specific fixed effects included.

Our results indicate that the average pretest score for male students is 9.3 points $\left(\beta_{0}\right)$, while for female students it is 6.4 points (i.e., $\beta_{0}+\beta_{2}$ ). Male students' post-test scores are, on average, 11.8 points $\left(\beta_{1}\right)$ higher than their pretest scores, while for female students the average improvement is 10.6 points (i.e., $\beta_{1}+\beta_{3}$ ). Thus, female students have lower pretest scores and also see smaller increases in performance from pretest to post-test, on average.

We can compare the raw coefficients from this analysis directly with the raw coefficients produced by the simple linear regressions in Eqs. (10a)-(10b) and the simple linear regression model for change scores:

$$
x_{\text {post }}-x_{\text {pre }}=\beta_{4}^{\prime}+\beta_{5}^{\prime} \text { Gender. }
$$

We summarize the relationship between MLR and mixed effects model coefficients in Table IV. Neither of the models represented by Eqs. (10b) and (14) control for students' pretest scores when modeling post-test scores or change scores; rather, these models estimate the average pretest scores, post-test scores, and change scores for students from different groups (i.e., males and females). Table IV indicates that these methods are identical.

We have not gone into the details of mixed effects models here, but there are several extensions of mixed effects models relevant to the current analysis. First: the random effects in the model may have a hierarchical structure. Here we have students nested within courses nested within different years, but the only significant structure to the errors was found to be the nesting of students within courses. For more detail on so-called "hierarchical linear modeling," see Ref. [4]. Furthermore, our random effects use the random-intercept form of linear mixed effects models, which assumes a different starting
TABLE IV. Quantitative comparison of simple linear regression coefficients [see Eqs. (10a)-(10b), (14)] and mixed effects coefficients [see Eq. (13)].

\begin{tabular}{lccr}
\hline \hline $\begin{array}{l}\text { Coefficient } \\
\text { interpretation }\end{array}$ & $\begin{array}{c}\text { MLR } \\
\text { coefficients }\end{array}$ & $\begin{array}{c}\text { Mixed effects } \\
\text { coefficients }\end{array}$ & Value \\
\hline $\begin{array}{c}\text { Average male } \\
\text { pretest score }\end{array}$ & $\beta_{0}^{\prime}$ & $\beta_{0}$ & 9.3 \\
$\begin{array}{c}\text { Average female } \\
\text { pretest score }\end{array}$ & $\beta_{0}^{\prime}+\beta_{1}^{\prime}$ & $\beta_{0}+\beta_{2}$ & 6.4 \\
$\begin{array}{c}\text { Average male } \\
\text { post-test score }\end{array}$ & $\beta_{2}^{\prime}$ & $\beta_{0}+\beta_{1}$ & 21.1 \\
$\begin{array}{c}\text { Average female } \\
\text { post-test score }\end{array}$ & $\beta_{2}^{\prime}+\beta_{3}^{\prime}$ & $\beta_{0}+\beta_{1}+\beta_{2}+\beta_{3}$ & 17.0 \\
$\begin{array}{c}\text { Average male } \\
\text { change score }\end{array}$ & $\beta_{4}^{\prime}$ & $\beta_{1}$ & 11.8 \\
$\begin{array}{c}\text { Average female } \\
\text { change score }\end{array}$ & $\beta_{4}^{\prime}+\beta_{5}^{\prime}$ & $\beta_{1}+\beta_{3}$ & 10.6 \\
\hline \hline
\end{tabular}

point for each student. Other mixed effects models compute random slopes, where the change in score over time would be different for different groups of students [5]. When accounting for the random effect of students, more than two time points are needed to model random slopes (otherwise there are zero degrees of freedom in the model). This would allow one to investigate whether there were significant variations in gains across courses or years in this sample. For a more complete treatment, see Ref. [5].

\section{CONCLUSION}

In the preceding sections, we have discussed how a researcher's choice of statistical methods and models ultimately depends on the question that is being investigated. Through an equity perspective, we have summarized the research questions about equity that various statistical methods can answer. We summarize this discussion in Table $\mathrm{V}$ and provide example models and scenarios that illustrate the utility of each method for answering different research questions.

The equity lens used in this paper, adapted from Refs. [3,20,21] provides an alternative perspective to ongoing debates in the PER community about the appropriateness of various statistical tools [1,2]. The choice of statistical tool and model ultimately depends on the research question being asked; comparisons of tools that address different research questions makes little sense. Researchers should make the goals of their study explicit and use appropriate methods to evaluate those goals.

Researchers should also evaluate whether assumptions are met for their choice of analysis tool (see Ref. [36]). Some of the assumptions described in the text for Cohen's $d$, MLR, and mixed effects models are rarely strictly satisfied in practice, but these tools can be robust to slightly violated assumptions [42-47]. Researchers should use 
TABLE V. Summary of research questions that can be answered about equity using the statistical tools discussed in this paper. We also include example models and scenarios that illustrate the utility of each method. Normalized gain or change does not fit within this paradigm of equity and cannot be interpreted in the same vein as these other statistics.

\begin{tabular}{|c|c|c|c|}
\hline Statistic & Research questions & Model & Example \\
\hline \multirow[t]{3}{*}{$\begin{array}{l}\text { Cohen's } d \\
\text { or ANOVA }\end{array}$} & Individuality & $d_{\text {paired }}\left(x_{\text {pre }}, x_{\text {post }}\right)$ & $\begin{array}{l}\text { Do female students improve in performance on the FCI, } \\
\text { on average, following instruction?; } d_{\text {paired }}>0\end{array}$ \\
\hline & Outcomes & $d\left(x_{\text {post }}^{\text {Group } 1}, x_{\text {post }}^{\text {Group2 }}\right)$ & $\begin{array}{l}\text { Do male (Group 1) and female (Group } 2 \text { ) students } \\
\text { have the same FCI post-test scores, on average?; } d=0\end{array}$ \\
\hline & Learning & $d\left(c_{\text {Group } 1}, c_{\text {Group } 2}\right)$ & $\begin{array}{l}\text { Do male (Group 1) and female (Group 2) students } \\
\text { have the same FCI change scores, on average?; } d=0\end{array}$ \\
\hline \multirow{2}{*}{$\begin{array}{l}\text { Normalized } \\
\text { gain or } \\
\text { change }\end{array}$} & Individuality & $g$ & Do female students improve in performance on FCI; $g>0$ \\
\hline & Opportunity* & \multicolumn{2}{|c|}{$\begin{array}{l}* \text { Normalized gain or change take pretest scores into account, } \\
\quad \text { but } g_{\text {Group } 1}=g_{\text {Group } 2} \text { does not indicate equity of opportunity. }\end{array}$} \\
\hline \multirow{5}{*}{$\begin{array}{l}\text { ANCOVA } \\
\text { or MLR }\end{array}$} & Individuality & $c=\beta_{0}$ & Do male students improve in performance on FCI; $\beta_{0}>0$ \\
\hline & Outcomes & $x_{\text {post }}=\beta_{0}+\beta_{1} \times$ Group & Do $\mathrm{M}$ and $\mathrm{F}$ students have the same post-test scores; $\beta_{1}=0$. \\
\hline & Learning & $c=\beta_{0}+\beta_{1} \times$ Group & Do $\mathrm{M}$ and $\mathrm{F}$ students have the same change in scores; $\beta_{1}=0$. \\
\hline & Opportunity & $\begin{aligned} x_{\mathrm{post}}= & \beta_{0}+\beta_{1} \times x_{\mathrm{pre}} \\
& +\beta_{2} \times \text { Group }\end{aligned}$ & $\begin{array}{l}\text { Do } \mathrm{M} \text { and } \mathrm{F} \text { students with the same pretest score } \\
\text { have the same predicted post-test (or change) score?; } \\
\beta_{2}=0\end{array}$ \\
\hline & & $\begin{aligned} c= & \beta_{0}+\beta_{1}^{\prime} \times x_{\mathrm{pre}} \\
& +\beta_{2} \times \text { Group }\end{aligned}$ & \\
\hline \multirow[t]{3}{*}{$\begin{array}{l}\text { Mixed } \\
\text { effects }\end{array}$} & Individuality & & $\begin{array}{l}\text { Do students improve in performance on the } \mathrm{FCI} \text {; } \\
\beta_{1}>0 \text { if reference group, } \beta_{1}+\beta_{3}>0 \text { otherwise. }\end{array}$ \\
\hline & Outcomes & & $\begin{array}{l}\text { Do male and female students have the same } \\
\text { post-test scores; } \beta_{2}+\beta_{3}=0 \text {. }\end{array}$ \\
\hline & Learning & $\begin{aligned} \text { Score }= & \beta_{0}+\beta_{1} \times \text { Test } \\
& +\beta_{2} \times \text { Group } \\
& +\beta_{3} \times \text { Test } \times \text { Group }\end{aligned}$ & $\begin{array}{l}\text { Do male and female students have the same } \\
\text { FCI change scores; } \beta_{3}=0 \text {. }\end{array}$ \\
\hline
\end{tabular}

caution when assumptions are grossly violated, however, as effect sizes and statistical significance levels will likely be biased. The statistical properties of normalized gain have not been rigorously studied. We examine the distribution of normalized gain in Ref. [36] and show that the mean and variance of normalized gain is undefined. We encourage other researchers to study the statistical properties of normalized gain, either analytically or numerically, to better understand this statistic.

We do not weigh in here on the value of different research questions, as these values likely differ among researchers. The results presented here should, however, allow researchers to more easily compare results obtained across studies with different research questions. Different results do not necessarily indicate disagreement between studies.

\section{ACKNOWLEDGMENTS}

We wish to thank Jeffrey Steinert for sharing data collected in his courses for this work. We thank Carl Wieman for extensive feedback on this manuscript, as well as Shima Salehi, Vince Coletta, Argenta Price, and Meagan Sundstrom for their thoughts on various versions of this manuscript. We thank two anonymous reviewers for their thoughtful comments on the organization and presentation of this manuscript.
[1] V. P. Coletta and J. J. Steinert, Why normalized gain should continue to be used in analyzing preinstruction and postinstruction scores on concept inventories, Phys. Rev. Phys. Educ. Res. 16, 010108 (2020).

[2] J. M. Nissen, R. M. Talbot, A. N. Thompson, and B. Van Dusen, Comparison of normalized gain and Cohen's $\mathrm{d}$ for analyzing gains on concept inventories, Phys. Rev. Phys. Educ. Res. 14, 010115 (2018).

[3] I. Rodriguez, E. Brewe, V. Sawtelle, and L. H. Kramer, Impact of equity models and statistical measures on interpretations of educational reform, Phys. Rev. ST Phys. Educ. Res. 8, 020103 (2012). 
[4] B. Van Dusen and J. Nissen, Modernizing use of regression models in physics education research: A review of hierarchical linear modeling, Phys. Rev. Phys. Educ. Res. 15, 020108 (2019).

[5] E. Theobald, Students are rarely independent: When, why, and how to use random effects in discipline-based education research, CBE Life Sci. Educ. 17, rm2 (2018).

[6] E. J. Theobald, M. Aikens, S. Eddy, and H. Jordt, Beyond linear regression: A reference for analyzing common data types in discipline based education research, Phys. Rev. Phys. Educ. Res. 15, 020110 (2019).

[7] R. R. Hake, Interactive-engagement versus traditional methods: A six-thousand-student survey of mechanics test data for introductory physics courses, Am. J. Phys. 66, 64 (1998).

[8] L. E. Kost, S. J. Pollock, and N. D. Finkelstein, Characterizing the gender gap in introductory physics, Phys. Rev. ST Phys. Educ. Res. 5, 010101 (2009).

[9] L. E. Kost-Smith, S. J. Pollock, and N. D. Finkelstein, Gender disparities in second-semester college physics: The incremental effects of a smog of bias, Phys. Rev. ST Phys. Educ. Res. 6, 020112 (2010).

[10] Z. Hazari, R. H. Tai, and P. M. Sadler, Gender differences in introductory university physics performance: The influence of high school physics preparation and affective factors, Sci. Educ. 91, 847 (2007).

[11] R. H. Tai and P. M. Sadler, Gender differences in introductory undergraduate physics performance: University physics versus college physics in the usa, Int. J. Sci. Educ. 23, 1017 (2001).

[12] A. Madsen, S. B. McKagan, and E. C. Sayre, Gender gap on concept inventories in physics: What is consistent, what is inconsistent, and what factors influence the gap?, Phys. Rev. ST Phys. Educ. Res. 9, 020121 (2013).

[13] S. L. Eddy and S. E. Brownell, Beneath the numbers: A review of gender disparities in undergraduate education across science, technology, engineering, and math disciplines, Phys. Rev. Phys. Educ. Res. 12, 020106 (2016).

[14] R. Henderson, J. Stewart, and A. Traxler, Partitioning the gender gap in physics conceptual inventories: Force Concept Inventory, Force and Motion Conceptual Evaluation, and conceptual survey of electricity and magnetism, Phys. Rev. Phys. Educ. Res. 15, 010131 (2019).

[15] R. Henderson, G. Stewart, J. Stewart, L. Michaluk, and A. Traxler, Exploring the gender gap in the conceptual survey of electricity and magnetism, Phys. Rev. Phys. Educ. Res. 13, 020114 (2017).

[16] V. P. Coletta, J. A. Phillips, and J. Steinert, FCI normalized gain, scientific reasoning ability, thinking in physics, and gender effects, AIP Conf. Proc. 1413, 23 (2012).

[17] M. Lorenzo, C. H. Crouch, and E. Mazur, Reducing the gender gap in the physics classroom, Am. J. Phys. 74, 118 (2006).

[18] S.D. Willoughby and A. Metz, Exploring gender differences with different gain calculations in astronomy and biology, Am. J. Phys. 77, 651 (2009).

[19] J. Day, J. B. Stang, N. G. Holmes, D. Kumar, and D. A. Bonn, Gender gaps and gendered action in a first-year physics laboratory, Phys. Rev. Phys. Educ. Res. 12, 020104 (2016).
[20] B. Van Dusen and J. Nissen, Equity in college physics student learning: A critical quantitative intersectionality investigation, J. Res. Sci. Teach. 57, 33 (2020).

[21] B. Van Dusen and J. Nissen, Associations between learning assistants, passing introductory physics, and equity: A quantitative critical race theory investigation, Phys. Rev. Phys. Educ. Res. 16, 010117 (2020).

[22] G. Smith and J. Smith, Regression to the mean in average test scores, Educational Assess. 10, 377 (2005).

[23] D. Hestenes, M. Wells, and G. Swackhamer, Force Concept Inventory, Phys. Teach. 30, 141 (1992).

[24] P. Eaton and S. Willoughby, Identifying a preinstruction to postinstruction factor model for the Force Concept Inventory within a multitrait item response theory framework, Phys. Rev. Phys. Educ. Res. 16, 010106 (2020).

[25] L. Ding and X. Liu, Getting started with quantitative methods in physics education research, in Getting Started in PER, 3rd ed. (AAPT, College Park, MD, 2012), Vol. 2.

[26] A. Madsen, E. Sayre, and S. McKagan, Effect size: What is it and when and how should I use it? (2016), https://www .physport.org/recommendations/Entry.cfm?ID=93385.

[27] J. J. McGough and S. V. Faraone, Estimating the size of treatment effects: Moving beyond $p$ values, Psychiatry (Edgmont) 6, 21 (2009).

[28] J. Cohen, Statistical Power Analysis for the Behavioral Sciences (Academic Press, New York, 2013).

[29] G. Glass and K. Hopkins, Statistical Methods in Education and Psychology (Pearson, 1996).

[30] B. K. Moser and G. R. Stevens, Homogeneity of variance in the two-sample means test, Am. Statistician 46, 19 (1992).

[31] J. M. Bland and D. G. Altman, Statistics notes: Some examples of regression towards the mean, Br. Med. J. 309, 780 (1994).

[32] A. G. Barnett, J. C. Van Der Pols, and A. J. Dobson, Regression to the mean: What it is and how to deal with it, Int. J. Epidemiol. 34, 215 (2005).

[33] S. Stephens and M. Marder, Longitudinal predictions using regression-corrected grouping to reduce regression to the mean, Phys. Rev. Phys. Educ. Res. 15, 020109 (2019).

[34] J. D. Marx and K. Cummings, Normalized change, Am. J. Phys. 75, 87 (2007).

[35] We note that "bias" as used in Ref. [34] indicates a correlation between pretest score and the effect size-in this case negative. This is different from the definition of statistical bias (e.g., $\mathbb{E}[s] \neq \sigma$ ), where the expectation value of a random variable from a sample is not equal to the true value of that variable for a population $\sigma$ [29].

[36] See Supplemental Material at http://link.aps.org/ supplemental/10.1103/PhysRevPhysEducRes.16.010141 for details on the mathematics and assumptions behind the models presented, as well as sample $\mathrm{R}$ code.

[37] J. H. Stock and M. W. Watson, Introduction to Econometrics (Pearson, Upper Saddle River, NJ, 2015).

[38] C. E. Werts and R. L. Linn, A general linear model for studying growth, Psychol. Bull. 73, 17 (1970).

[39] P. D. Allison, Change scores as dependent variables in regression analysis, Sociol. Methodol. 20, 93 (1990). 
[40] K. P. Burnham and D. R. Anderson, Model Selection and Multimodel Inference (Springer Verlag, New York, 2002), Vol. 20, pp. 93-114.

[41] A. Kuznetsova, P. B. Brockhoff, and R. H. B. Christensen, lmerTest package: Tests in linear mixed effects models, J. Stat. Softw. 82, 1 (2017).

[42] T. Heeren and R. D'Agostino, Robustness of the two independent samples $t$ test when applied to ordinal scaled data, Stat. Med. 6, 79 (1987).

[43] S. S. Sawilowsky and R. Clifford Blair, A more realistic look at the robustness and type ii error properties of the $\mathrm{t}$ test to departures from population normality., Psychol. Bull. 111, 352 (1992).
[44] D.W. Zimmerman, Two separate effects of variance heterogeneity on the validity and power of significance tests of location, Stat. Methodol. 3, 351 (2006).

[45] B. L. Welch, On the comparison of several mean values: An alternative approach, Biometrika 38, 330 (1951).

[46] T. Lumley, P. Diehr, S. Emerson, and L. Chen, The importance of the normality assumption in large public health data sets, Annu. Rev. Public Health 23, 151 (2002).

[47] D. A. Belsley, E. Kuh, and R. E. Welsch, Regression Diagnostics: Identifying Influential Data and Sources of Collinearity (John Wiley \& Sons, New York, 2005), Vol. 571. 